\title{
Unsupervised Distillation of Syntactic Information from Contextualized Word Representations
}

\author{
Shauli Ravfogel ${ }^{* 1,3}$ Yanai Elazar* 1,3 Jacob Goldberger ${ }^{2}$ Yoav Goldberg ${ }^{1,3}$ \\ ${ }^{1}$ Computer Science Department, Bar Ilan University \\ ${ }^{2}$ Faculty of Engineering, Bar Ilan University \\ ${ }^{3}$ Allen Institute for Artificial Intelligence \\ \{shauli.ravfogel, yanaiela,yoav.goldberg\}@gmail.com \\ jacob.goldberger@biu.ac.il
}

\begin{abstract}
Contextualized word representations, such as ELMo and BERT, were shown to perform well on various semantic and syntactic task. In this work, we tackle the task of unsupervised disentanglement between semantics and structure in neural language representations: we aim to learn a transformation of the contextualized vectors, that discards the lexical semantics, but keeps the structural information. To this end, we automatically generate groups of sentences which are structurally similar but semantically different, and use metric-learning approach to learn a transformation that emphasizes the structural component that is encoded in the vectors. We demonstrate that our transformation clusters vectors in space by structural properties, rather than by lexical semantics. Finally, we demonstrate the utility of our distilled representations by showing that they outperform the original contextualized representations in a few-shot parsing setting.
\end{abstract}

\section{Introduction}

Human language ${ }^{1}$ is a complex system, involving an intricate interplay between meaning (semantics) and structural rules between words and phrases (syntax). Self-supervised neural sequence models for text trained with a language modeling objective, such as ELMo (Peters et al., 2018), BERT (Devlin et al., 2019), and RoBERTA (Liu et al., 2019b), were shown to produce representations that excel in recovering both structure-related information (Gulordava et al., 2018; van Schijndel and Linzen, 2018; Wilcox et al., 2018; Goldberg, 2019) as well as in semantic information (Yang et al., 2019; Joshi et al., 2019).

In this work, we study the problem of disentangling structure from semantics in neural language

\footnotetext{
${ }^{*}$ Equal contribution

${ }^{1}$ In this work we focus on English.
}

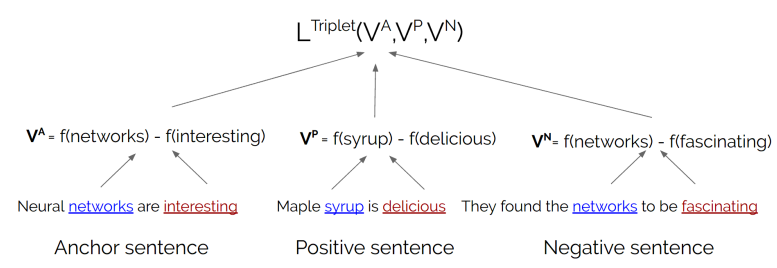

Figure 1: An illustration of triplet-loss calculation. Pairs of words are represented by the difference between their transformation $f$, which is identical for all words. The pairs of words in the anchor and positive sentences are lexically different, but structurally similar. The negative example presented here is especially challenging, as it is lexically similar, but structurally different.

representations: we aim to extract representations that capture the structural function of words and sentences, but which are not sensitive to their content. For example, consider the sentences:

1. Neural networks are interesting.

2. I study neural networks.

3. Maple syrup is delicious.

4. John loves maple syrup.

While (1) and (3) are different in content, they share a similar structure, the corresponding words in them, while unrelated in meaning, ${ }^{2}$ serve the same function. Similarly for sentences (2) and (4). In contrast, sentence (1) shares the phrase neural networks with sentence (2), and maple syrup is shared between (3) and (4). ${ }^{3}$ While the two occurrences of each phrase share the meaning, they are used in different structural (syntactic) configurations, serving different roles within the sentence

\footnotetext{
${ }^{2}$ We focus on lexical semantics.

${ }^{3}$ There is a syntactic distinction between the two, with "maple" being part of a noun compound and "neural" being an adjective. However, we focus in their similarity as noun modifiers in both phrases.
} 
(appearing in subject vs object position). ${ }^{4}$ We seek a representation that will expose the similarity between "networks" in (1) and "syrup" in (2), while ignoring the similarity between "syrup" in (2) and "syrup" in (4).

We seek a function from contextualized word representations to a space that exposes these similarities. Crucially, we aim to do this in an unsupervised manner: we do not want to inform the process of the kind of structural information we want to obtain. We do this by learning a transformation that attempts to remove the lexical-semantic information in a sentence, while trying to preserve structural properties.

Disentangling syntax from lexical semantics in word representations is a desired property for several reasons. From a purely scientific perspective, once disentanglement is achieved, one can better control for confounding factors and analyze the knowledge the model acquires, e.g. attributing the predictions of the model to one factor of variation while controlling for the other. In addition to explaining model predictions, such disentanglement can be useful for the comparison of the representations the model acquires to linguistic knowledge. From a more practical perspective, disentanglement can be a first step toward controlled generation/paraphrasing that considers only aspects of the structure, akin to the style-transfer works in computer vision, i.e., rewriting a sentence while preserving its structural properties while ignoring its meaning, or vice-versa. It can also inform searchbased application in which one can search for "similar" texts while controlling various aspects of the desired similarity.

To achieve this goal, we begin with the intuition that the structural component in the representation (capturing the form) should remain the same regardless of the lexical semantics of the sentence (the meaning). Rather than beginning with a parsed corpus, we automatically generate a large number of structurally-similar sentences, without presupposing their formal structure $(\S 3.1)$. This allows us to pose the disentanglement problem as a metriclearning problem: we aim to learn a transformation of the contextualized representation, which is invariant to changes in the lexical semantics within each group of structurally-similar sentences (§3.3). We demonstrate the structural properties captured

\footnotetext{
${ }^{4}$ These differences in syntactic position are also of relevance to language modeling, as different positions may pose different restrictions on the words that can appear in them.
}

by the resulting representations in multiple experiments ( $(4)$, among them automatic identification of structurally-similar words and few-shot parsing.

We release our code at https://github.com/ shauli-ravfogel/NeuralDecomposition.

\section{Related Work}

The problem of disentangling different sources of variation has long been studied in computer vision, and was recently applied to neural models (Bengio et al., 2013; Mathieu et al., 2016; Hadad et al., 2018). Such disentanglement can assist in learning representations that are invariant to specific factors, such as pose-invariant face-recognition (Peng et al., 2017) or style-invariant digit recognition (Narayanaswamy et al., 2017). From a generative point of view, disentanglement can be used to modify one aspect of the input (e.g., "style"), while keeping the other factors (e.g., "content") intact, as done in neural image style-transfer (Gatys, 2017).

In NLP, disentanglement is much less researched. In controlled natural language generation and style transfer, several works attempted to disentangle factors of variation such as sentiment or age of the writer, with the intention to control for those factors and generate new sentences with specific properties (Sohn et al., 2015; Ficler and Goldberg, 2017; Lample et al., 2018), or transfer existing sentences to similar sentences that differ only in the those properties. The latter goal of style transfer is often realized by learning representations which are invariant to the controlled attributes (Fu et al., 2018; Hu et al., 2017).

Another main line of work which is relevant to our approach is that of probing. The concept, originally introduced by Adi et al. (2016) and Hupkes et al. (2018), relies on training classifiers (probes) to expose symbolic linguistic information that is encoded in the model. A large body of works have shown sensitivity to both semantic (Tenney et al., 2019a; Richardson et al., 2019) and syntactic (Tenney et al., 2019b; Lin et al., 2019; Reif et al., 2019; Hewitt and Manning, 2019; Liu et al., 2019a) information. Hewitt and Manning (2019) demonstrated that it is possible to train a linear transformation, under which squared euclidean distance between transformed contextualized word vectors correspond to the distances between the respective words in the syntactic tree. Li and Eisner (2019) have used a variational estimation method (Alemi et al., 2016) of the information-bottleneck principle 
(Tishby et al., 1999) to extract word embeddings that are useful to the end task of parsing.

While impressive, those works presuppose a specific syntactic structure (e.g. annotated parse tree) and use this linguistic signal to learn the probe in a supervised manner. This approach can introduce confounding between extracting information and learning it by the probe (Hewitt and Liang, 2019; Ravichander et al., 2020; Maudslay et al., 2020; Elazar et al., 2020). In contrast, we aim to expose the structural information encoded in the network in an unsupervised manner, without pre-supposing an existing syntactic annotation scheme.

\section{Method}

Our goal is to learn a function $f: \mathbb{R}^{n} \mapsto \mathbb{R}^{m}$, which operates on contextualized word representations $x$ and extracts vectors $f(x)$ which make the structural information encoded in $x$ more salient, while discarding as much lexical information as possible. In the sentences "Maple syrup is delicious" and "Neural networks are interesting", we want to learn a function $f$ such that $f\left(v_{\text {syrup }}^{3}\right) \approx$ $f\left(v_{\text {networks }}^{1}\right)$, where $v_{\text {word }}^{i}$ is the contextualized vector representation of the word in sentence $i$. We also want $f\left(v_{\text {syrup }}^{4}\right) \approx f\left(v_{\text {networks }}^{2}\right)$, while keeping $f\left(v_{\text {networks }}^{1}\right) \not \approx f\left(v_{\text {networks }}^{2}\right)$.

Moreover, we would like the relation between the words "maple" and "delicious" in the third sentence, to be similar to the relation between "neural" and "interesting" in the first sentence: $\operatorname{pair}\left(v_{\text {maple }}^{3}, v_{\text {delicious }}^{3}\right) \approx \operatorname{pair}\left(v_{\text {neural }}^{1}, v_{\text {interesting }}^{1}\right)$. Operatively, we represent pairs of words $(x, y)$ by the difference between their transformation $f(x)-$ $f(y)$, and aim to learn a function $f$ that preserves: $f\left(v_{\text {maple }}^{3}\right)-f\left(v_{\text {delicious }}^{3}\right) \approx f\left(v_{\text {neural }}^{1}\right)-f\left(v_{\text {interesting }}^{1}\right)$. The choice to represent pairs this way was inspired by several works that demonstrated that nontrivial semantic and syntactic relations between uncontextualized word representations can be approximated by simple vector arithmetic (Mikolov et al., 2013a,b; Levy and Goldberg, 2014).

To learn $f$, we start with groups of sentences such that the sentences within each group are known to share structure but differ in lexical semantics. We call the sentences in each group structurally equivalent. Figure 2 shows an example of two structurally equivalent sets. Acquiring such sets is challenging, especially if we do not assume a known syntactic formalism and cannot mine for sentences based on their observed tree structures.
To this end, we automatically generate the sets starting with known sentences and sampling variants from a language model ( $\$ 3.1)$. Our sentence-set generation procedure ensures that words from the same set that share an index also share their structural function. We call such words corresponding.

We now proceed to learn a function $f$ to map contextualized vectors of corresponding words (and the relations between them, as described above) to neighbouring points in the space.

We train $f$ such that the representation assigned to positive pairs - pairs that share indices and come from the same equivalent set - is distinguished from the representations of negative pairs - challenging pairs that come from different sentences, and thus do not share the structure of the original pair, but can, potentially, share their lexical meaning. We do so using Triplet loss, which pushes the representations of pairs coming from the same group closer together (§3.3). Figure 1 sketches the network.

\subsection{Generating Structurally-similar Sentences}

In order to generate sentences that approximately share their structure, we sequentially replace content words in the sentence with other content words, while aiming to maintain the grammatically of the sentence, and keep its structure intact.

Since we do not want to rely on syntactic annotation when performing this replacement, we opted to use a pre-trained language model - BERT - under the assumption that strong neural language models do implicitly encode many of the syntactic restrictions that apply to words in different grammatical functions (e.g., we assume that BERT would not predict a transitive verb in the place of an intransitive verb, or a verb that accepts a complement in the place of a verb that does not accept a complement). While this assumption seems to hold with regard to basic distinctions such as transitive vs. intransitive verbs, its validity is less clear in the more nuanced cases, in which small differences in the surface level can translate to substantial differences in abstract syntactic structure - such as replacing a control verb with a raising verb. This is a limitation of the current approach, although we find that the average sentence we generate is grammatical and similar in structure to the original sentence. Moreover, as our goal is to expose the structural similarity encoded in neural language models, we 
- When a train ticket is purchased, a contract is established

- When a travel document is acquired, a settlement is declared

- When a winning vehicle is obtained, a competition is introduced

- When a winning bid is announced, a winner is created
- Shapley participated in the "great debate " with heber d

- Khan joined in the " silent discussion "with e t

- Parker figured in the " coming showdown "with block leader

- Moore engaged in the " modern struggle "with joseph israel

Figure 2: Two groups of structurally-equivalent sentences. In each group, the first sentence is original sentence from Wikipedia, and the sentences below it were generated by the process of repeated BERT substitution. Some sets of corresponding words-that is, words that share the same structural function-are highlighted in the same color.

find it reasonable to only capture the distinctions that are captured by modern language models.

Implementation We start each group with a Wikipedia sentence, for which we generate $k=6$ equivalent sentences by iterating over the sentence from left to right sequentially, masking the ith word, and replacing it with one of BERT's top-30 predictions. To increase semantic variability, we perform the replacement in place (online): after randomly choosing a guess $w$, we insert $w$ to the sentence at index $i$, and continue guessing the $i+1$ word based on the modified sentence. ${ }^{5}$ We exclude a closed set of a few dozens of words (mostly function words) and keep them unchanged in all $k$ variations of a sentence. We further maintain structural correctness by maintaining the $\operatorname{POS}^{6}$, and encourage semantic diversity by the auto-regressive replacement process. In Table 6 in the Appendix we show some additional generated groups. The sets in Figure 2 were generated using this method.

\subsection{Word Representation}

We sample $N=150,000$ random sentences and use the our method to generate 900,000 equivalent sets $E$ of structurally equivalent sentences. Then, we encode the sentences and randomly collect 1, 500, 000 contextualized vector representations of words from these sets, resulting in 1,500,000 training pairs and 200,000 evaluation pairs for the training process of $f$. We experiment with both ELMo and BERT language models. In average, we sample 11 word-pairs from each group of equivalent sentences. For ELMo, we represent each word in context as a concatenation of the last two ELMo layers (excluding the word embedding layer, which is not contextualized and therefore irrelevant for

\footnotetext{
${ }^{5}$ We note that this process bears some similarity to Gibbs sampling from BERT conditioned LM.

${ }^{6}$ We maintain the same POS so that the dataset will be valid for other tasks that require structure-preserving variants. However, In practice, we did not observe major differences when repeating the experiments reported here without the POS-preserving constraint when generating the data.
}

structure), resulting in representations of dimension 2048. For BERT, we concatenate the mean of the words' representation ${ }^{7}$ across all contextualized layers of BERT-Large, with the representation of layer 16, which was found by Hewitt and Manning (2019) most indicative of syntax.

\subsection{Triplet Loss}

We learn the mapping function $f$ using triplet loss (Figure 1). Given a group of equivalent sentences $E_{i}$, we randomly choose two sentences to be the anchor sentence $S^{A}$ and the positive sentence $S^{P}$, and sample two different word indices $\left\{i_{1}, i_{2}\right\}$. Let $S^{A}\left[i_{1}\right]$ be the contextualized representation of the $i_{1}$ th word in sentence $S^{A}$. The words $S^{A}\left[i_{1}\right]$ and $S^{A}\left[i_{2}\right]$ from the anchor sentence would form a representation of a pair of words, which should be close to the pair $S^{P}\left[i_{1}\right], S^{P}\left[i_{2}\right]$ from the positive sentence.

We represent pairs as their differences after transformation, resulting in the anchor pair $V^{A}$ and positive pair $V^{P}$ :

$$
\begin{aligned}
V^{A} & =f\left(S^{A}\left[i_{1}\right]\right)-f\left(S^{A}\left[i_{2}\right]\right) & & S^{A} \in E_{i} \\
V^{P} & =f\left(S^{P}\left[i_{1}\right]\right)-f\left(S^{P}\left[i_{2}\right]\right) & & S^{P} \in E_{i}
\end{aligned}
$$

where $f$ is the parameterized syntactic transformation we aim to learn. We also consider a negative pair:

$$
V^{N}=f\left(S^{N}\left[j_{1}\right]\right)-f\left(S^{N}\left[j_{2}\right]\right) \quad S^{N} \notin E_{i}
$$

coming from sentence $S^{N}$ which is not in the equivalent set.

As $f$ has shared parameters for both words in the pair, it can be considered a part of a Siamese network, making our learning procedure an instance of a triplet Siamese network (Schroff et al., 2015). We choose $f$ to be a simple model: a single linear layer that maps from dimensionality 2048 to 75 .

\footnotetext{
${ }^{7}$ Since BERT uses word-piece tokenization, we take the first token to represent each word.
} 
The dimensions of the transformation were chosen according to development set performance.

We use triplet loss (Schroff et al., 2015) to move the representation of the anchor vector $V^{A}$ closer to the representation of the positive vector $V^{P}$ and farther apart from the representation of the negative vector $V^{N}$. Following Hoffer and Ailon (2015), we calculate the softmax version of the triplet loss:

$$
L^{\text {triplet }}\left(V^{A}, V^{P}, V^{N}\right)=\frac{e^{d\left(V^{A}, V^{P}\right)}}{e^{d\left(V^{A}, V^{P}\right)}+e^{d\left(V^{A}, V^{N}\right)}}
$$

where $d(x, y)=1-\frac{x^{\top} y}{\|x\|\|y\|}$ is the cosinedistance between the vectors $x$ and $y$. Note that $L^{\text {triplet }} \rightarrow 0$ as $\frac{d\left(V^{A}, V^{P}\right)}{d\left(V^{A}, V^{N}\right)} \rightarrow 0$, as expected. The triplet objective is optimized end-to-end using the Adam optimizer (Kingma and Ba, 2015). We train for 5 epochs with a mini-batch of size $500^{8}$, and take the last model as the final syntactic extractor. During training, the gradient backpropagates through the pair vectors to the parameters $f$ of the Siamese model, to get representations of individual words that are similar for corresponding words in equivalent sentences. We note that we do not back-propagate the gradient to the contextualized vectors: we keep them intact, and only adjust the learned transformation.

Hard negative sampling We obtain the negative vectors $V^{N}$ using hard negative sampling. For each mini-batch $B$, we collect $500\left\{\mathrm{~V}_{i}^{A}, \mathrm{~V}_{i}^{P}\right\}$ pairs, each pair taken from an equivalent set $E_{i}$. The negative instances $\mathrm{V}_{i}^{N}$ are obtained by searching the batch for a vector that is closest to the anchor and comes from a different set:

$$
V_{i}^{N}=\underset{V_{j \neq i}^{A} \in B}{\arg \min } d\left(V_{i}^{A}, V_{j}^{A}\right) .
$$

In addition, we enforce a symmetry between the anchor and positive vectors, by adding a pair (positive, anchor) for each pair (anchor, positive) in $B$. That is, $V_{i}^{N}$ is the "most misleading" word-pair vector: it comes from a sentence that has a different structure than the structure of $\mathrm{V}_{i}^{A}$ sentence, but is the closest to $\mathrm{V}_{i}^{A}$ in the mini-batch.

\section{Experiments and Analysis}

We have trained the syntactic transformation $f$ in a way that should encourage it to retain the structural

\footnotetext{
${ }^{8} \mathrm{~A}$ large enough mini-batch is necessary to find challenging negative examples.
}

information encoded in contextualized vectors, but discard other information. We assess the representations the model acquired in an unsupervised manner, by evaluating the extent to which the local neighbors of each transformed contextualized vector $f(x)$ share known structural properties, such as grammatical function within the sentence. For the baseline, we expect the neighbors of each vector to share a mix of semantic and syntactic properties. For the transformed vectors, we expect the neighbors to share mainly syntactic properties. Finally, we demonstrate that in a few-shot setting, our representations outperform the original ELMO representation, indicating they are indeed distilled from syntax, and discard other information that is encoded in ELMO vectors but is irrelevant for the extraction of the structure of a sentence.

Corpus For training the transformation $f$, we rely on 150,000 sentences from Wikipedia, tokenized and POS-tagged by spaCy (Honnibal and Johnson, 2015; Honnibal and Montani, 2017). The POS tags are used in the equivalent set generation to filter replacement words. Apart from POS tagging, we do not rely on any syntactic annotation during training. The evaluation sentences for the experiments mentioned below are sampled from a collection of 1,000,000 original and unmodified Wikipedia sentences (different from those used in the model training).

\subsection{Qualitative Analysis}

t-SNE Visualization Figure 3 shows a 2dimensional t-SNE projection (Maaten and Hinton, 2008) of 15,000 random content words. The left panel projects the original ELMo states, while the right panel is the syntactically transformed ones. The points are colored according to the dependency label (relation to parent) of the corresponding word, predicted by the parser.

In the original ELMo representation most states - apart from those characterized by a specific partof-speech, such as amod (adjectives, in orange) or nummod (numbers, in light green) - do not fit well into a single cluster. In contrast, the syntactically transformed vectors are more neatly clustered, with some clusters, such as direct objects (brown) and prepositional-objects (blue), that are relatively separated after, but not before, the transformation. Interestingly, some functions that used to be a single group in ELMo (like the adjectives in orange, or the noun-compounds in green) are 


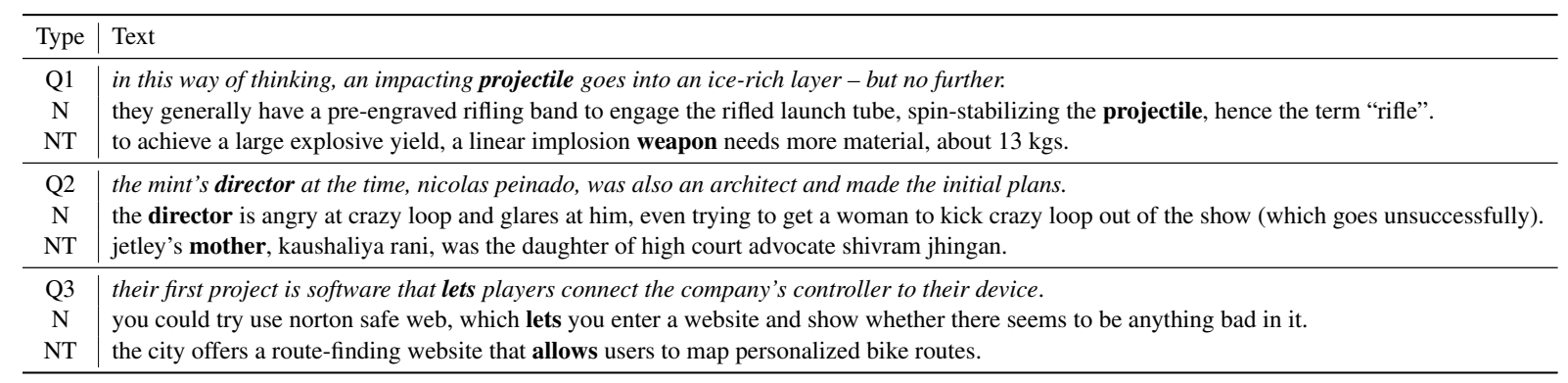

Table 1: Text examples for a few query words (in the Q rows, in bold), and their closest neighbours before (N) and after (NT) the transformation.
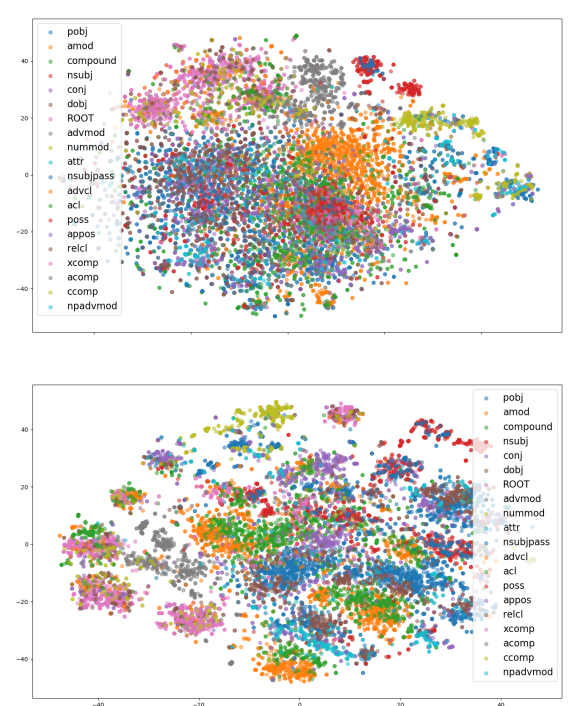

Figure 3: t-SNE projection of ELMO states, colored by syntactic function, before (upper) and after (lower) the syntactic transformation.

now split into several clusters, corresponding to their use in different sentence positions, separating for examples adjectives that are used in subject positions from those in object position or within prepositional phrases. Additionally, as noun compounds ("maple" in "maple syrup") and adjectival modifiers ("tasty" in "tasty syrup") are relatively structurally similar (they appear between determiners and nouns within noun phrases, and can move with the noun phrase to different positions), they are split and grouped together in the representation (the green and orange clouds).

To quantify the difference, we run $K$-means clustering on the projected vectors, and calculate the average cluster purity score as the relative proportion of the most common dependency label in each cluster. The higher this value is, the more the division to clusters reflect division to grammatical functions (dependency labels). We run the clustering with different $K$ values: $10,20,40,80$. We find an increase in class purity following our transformation: from scores of $22.6 \%, 26.8 \%, 32.6 \%$ and $36.4 \%$ (respectively) for the original vectors, to scores of $24.3 \%, 33.4 \%, 42.1 \%$ and $48.0 \%$ (respectively) for the transformed vectors.

Examples In Table 1 we present a few query words $(\mathrm{Q})$ and their closest neighbours before $(\mathrm{N})$ and after (NT) the transformation. Note the high structural similarity of the entire sentence, as well as the function of the word within it (Q1: last word of subject NP in a middle clause, Q2: possessed noun in sentence initial subject NP, Q3: head of relative clause of a direct object).

Additional examples (including cases in which the retrieved vector does not share the dependency edge with the query vector) are supplied in Appendix $\S$ A.

\subsection{Quantitative Evaluation}

We expect the transformed vectors to capture more structural and less lexical similarities than the source vectors. We expect each vectors' neighbors in space to share the structural function of the word over which the vector was collected, but not necessarily share its lexical meaning. We focus on the following structural properties: (1) Dependencytree edge of a given word (dep-edge), that represents its function (subject, object etc.). (2) The dependency edge of the word parent's (head's depedge) in the tree - to represent higher level structure, such as a subject that resides within a relative clause, as in the word "man" in the phrase "the child that the man saw". (3) Depth in the dependency tree (distance from the root of the sentence tree). (4) Constituency-parse paths: consider, for example, the sentence "They saw the moon with the telescope". The word "telescope" is a part of a 


\begin{tabular}{lccccccc}
\hline & Dep. edge & Head's dep. edge & $\begin{array}{c}\text { Tree path } \\
\text { (complete) }\end{array}$ & $\begin{array}{c}\text { Tree path } \\
(\mathrm{L}=3)\end{array}$ & $\begin{array}{c}\text { Tree path } \\
(\mathrm{L}=2)\end{array}$ & $\begin{array}{c}\text { Depth } \\
\text { (correlation) }\end{array}$ & $\begin{array}{c}\text { Lexical Match } \\
\text { Baseline (all) }\end{array}$ \\
Transformed (all) & 0.580 & 0.473 & 0.166 & 0.353 & 0.566 & 0.448 & 0.736 \\
\hline Transformed-untrained (all) & 0.461 & 0.603 & 0.253 & 0.523 & 0.735 & 0.561 & 0.284 \\
\hline Baseline (hard) & 0.509 & 0.430 & 0.142 & 0.319 & 0.528 & 0.407 & 0.680 \\
Transformed (hard) & 0.671 & 0.591 & 0.160 & 0.347 & 0.564 & 0.430 & 0.776 \\
\hline
\end{tabular}

Table 2: Closest-word queries, before and after the application of the syntactic transformation. "Basline" refers to unmodified ELMo vectors, "Transformed" refers to ELMo vectors after the learned syntactic transformation $f$, and "Transformed-untrained" refers to ElMo vectors, after a transformation that was trained on a randomly-initialized ELMo. "hard" denotes results on the subset of POS tags which are most structurally diverse.

noun-phrase "the telescope", which resides inside a prepositional phrase "with the telescope", which is part of the Verbal phrase "saw with the telescope". The complete constituency path for this word is therefore "NP-PP-VP". We calculate the complete tree path to the root (Tree-path-complete), as well as paths limited to lengths 2 and 3 .

For this evaluation, we parse 400,000 random sentences taken from the 1-million-sentences Wikipedia sample, run ELMo and BERT to collect the contextualized representations of the sentences, and randomly choose 400,000 query word vectors (excluding function words). We then retrieve, for each query vector $x$, the value vector $y$ that is closest to $x$ in cosine-distance, and record the percentage of closest-vector pairs $(x, y)$ that share each of the structural properties listed above. For the tree depth property, we calculate the Pearson correlation between the depths of the queries and the retrieved values. We use the Berkeley Neural Parser (Kitaev and Klein, 2018) for constituency parsing. We exclude function words from the evaluation.

Easier and Harder cases The baseline models tend to retrieve words that are lexically similar. Since certain words tend to appear at above-chance probability in certain structural functions, this can make the baseline be "right for the wrong reason", as the success in the closest-word test reflects lexical similarity, rather than grammatical generalization. To control for this confounding, we sort the different POS tags according to the entropy of their dependency-labels distribution, and repeat the evaluation only for words belonging to those POS tags having the highest entropy (those are the most structurally variant, and tend to appear in different structural functions). The performance of the baselines (ELMo, BERT models) on those words drops significantly, while the performance of our model is only mildly influenced, indicating the superiority of the model in capturing structural rather than lexical information.

Results The results for ELMo are presented in Table 2. For BERT, we witnessed similar, but somewhat lower, accuracy: for example, $68.1 \%$ dependency-edge accuracy, $56.5 \%$ head's dependency-edge accuracy, and $22.1 \%$ complete constituency-path accuracy. The results for BERT are available in Appendix $\S \mathrm{B}$, and for the reminder of the paper, we focus in ELMo. We observe significant improvement over the baseline for all tests. The correlation between the depth in tree of the query and the value words, for examples, rises from $44.8 \%$ to $56.1 \%$, indicating that our model encourages the structural property of the depth of the word to be more saliently encoded in its representation compared with the baseline. The most notable relative improvement is recorded with regard to full constituency-path to the root: from $16.6 \%$ before the structural transformation, to $25.3 \%$ after it - an improvement of $52 \%$. In addition to the increase in syntax-related properties, we observe a sharp drop - from $73.6 \%$ to $28.4 \%$ - in the proportion of query-value pairs that are lexically identical (lexical match, Table 2). This indicates our transformation $f$ removes much of the lexical information, which is irrelevant for structure. To assess to what extent the improvements stems from the information encoded in ELMo, rather than being an artifact of the triplet-loss training, we also evaluate on a transformation $f$ that was trained on a randomlyinitialized ELMo, a surprisingly strong baseline (Conneau et al., 2018). We find this model performs substantially worse than the baseline (Table 2, "Transformed-untrained (all)").

\subsection{Minimal Supervision for Structure Distillation: Few-Shot Parsing}

The absolute nearest-neighbour accuracy values may appear to be relatively low: for example, only $67.6 \%$ of the (query, value) pairs share the same 

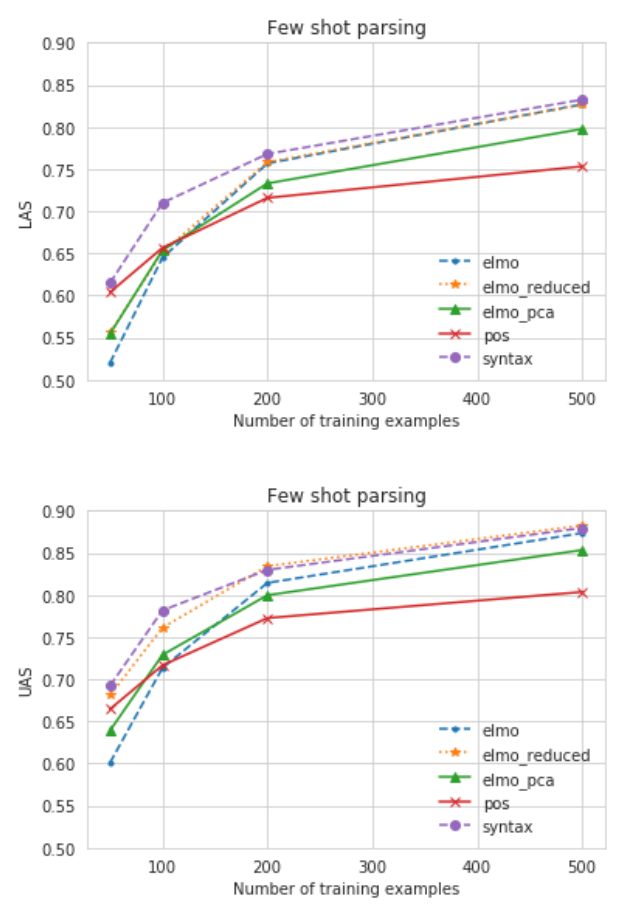

Figure 4: Results of the few-shots parsing setup.

dependency edge.

As the model acquires its representation without being exposed to human-mandated syntactic convention, some of the apparent discrepancies in nearest neighbours may be due to the fact the model acquires different kind of generalization, or learned a representation that emphasizes different kinds of similarities. Still, we expect the resulting (75 dimensional) representations to contain distilled structure information that is mappable to human notions of syntax. To test this, we compare dependency-parsers trained on our representation and on the source representation. If our representation indeed captures structural information, we expect it to excel on a low data setting. To this end, we test our hypothesis with few-shot dependency parsing setup, where we train a model to predict syntactic trees representation with only a few hundred labeled examples.

We use an off-the-shelf dependency parser model (Dozat and Manning, 2016) and swap the pre-trained Glove embeddings (Pennington et al., 2014) with ELMo contextualized embeddings (Peters et al., 2018). In order to have a fair comparison with our method, we use the concatenation of the two last layers of Elmo; we refer to this experiment as elmo. As our representation is much smaller than ELMo's (75 as opposed to 2048), a potential issue for a low data setting is the higher number of parameters to optimize in the later case, therefore a lower dimension may achieve better results. We design two additional baselines to remedy this potential issue: (1) Using PCA in order to reduce the representation dimensionality. We randomly chose $1 \mathrm{M}$ words from Wikipedia, calculated their representation with ELMo embeddings and performed PCA. This transformation is applied during training on top of ELMo representation while keeping the 75 first components. This experiment is referred to as elmo-pca. This representation should perform well if the most salient information in the ELMo representations are structural. We exepct it to not be the case. (2) Automatically learning a matrix that reduces the embedding dimension. This matrix is learned during training and can potentially extract the relevant structural information from the representations. We refer to this experiment as elmo-reduced. Additionally, we also compare to a baseline where we use the gold-POS labels as the sole input to the model, by initializing an embedding matrix of the same size for each POS. We refer to this experiment as pos. Lastly, we examine the performance of our representation, where we apply our structural extraction method on top of ELMo representation. We refer to this experiment as syntax.

We run the few-shot setup with multiple training size values: 50, 100, 200, 500. The results-for both labeled (LAS) and unlabeled (UAS) attachment scores-are presented in Figure 4, and the numerical results are available in the Appendix $\S \mathrm{C}$. In the lower training size setting, we obtain the best performances compared to all baselines. The more training data is used, the gap between our representation and the baselines reduced, but the syntax representation still outperforms elmo. Using gold POS labels as inputs works relatively well with 50 training examples, but it quickly reaches a plato in performance and remains behind the other baselines. Reducing the dimensions with PCA (elmo-pca) works considerably worse than ELMo, indicating PCA loses important information. Reducing the dimensions with a learned matrix (elmo-reduced) works substantially better than ELMo, and achieve the same UAS as our representation from 200 training sentences onward. However, our transformation was learned in an unsupervised fashion, without access to the syntactic trees. 
Finally, when considering the labeled attachment score, where the model is tasked at predicting not only the child-parent relation but also its label, our syntax representation outperforms elmo-reduced.

\section{Conclusion}

We propose an unsupervised method for the distillation of structural information from neural contextualized word representations. We used a process of sequential BERT-based substitution to create a large number of sentences which are structurally similar, but semantically different. By controlling for structure while changing lexical choice, we learn a metric under which pairs of words that come from structurally-similar sentences are close in space. We demonstrated that the representations acquired by this method share structural properties with their neighbors in space, and show that with a minimal supervision, those representations outperform ELMo in the task of few-shots parsing. The method is a first step towards a better disentanglement between various kinds of information that is represented in neural sequence models.

The method used to create the structurally equivalent sentences can be useful by its own as a dataaugmentation technique. In future work, we aim to extend this method to allow for a more soft alignment between structurally-equivalent sentences.

\section{Acknowledgments}

We would like to thank Gal Chechik for providing valuable feedback on early version of this work. This project has received funding from the Europoean Research Council (ERC) under the Europoean Union's Horizon 2020 research and innovation programme, grant agreement No. 802774 (iEXTRACT). Yanai Elazar is grateful to be partially supported by the PBC fellowship for outstanding $\mathrm{PhD}$ candidates in Data Science.

\section{References}

Yossi Adi, Einat Kermany, Yonatan Belinkov, Ofer Lavi, and Yoav Goldberg. 2016. Fine-grained analysis of sentence embeddings using auxiliary prediction tasks. CoRR, abs/1608.04207.

Alexander Alemi, Ian Fischer, Joshua V. Dillon, and Murphy Murphy. 2016. Deep variational information bottleneck. In Proceedings of the International Conference on Learning Representations (ICLR).

Yoshua Bengio, Aaron C. Courville, and Pascal Vincent. 2013. Representation learning: A review and new perspectives. IEEE Trans. Pattern Anal. Mach. Intell., 35(8):1798-1828.

Alexis Conneau, Germán Kruszewski, Guillaume Lample, Loïc Barrault, and Marco Baroni. 2018. What you can cram into a single $\backslash \$ \&$ ! \#* vector: Probing sentence embeddings for linguistic properties. In Proceedings of the 56th Annual Meeting of the Association for Computational Linguistics, ACL 2018, Melbourne, Australia, July 15-20, 2018, Volume 1: Long Papers, pages 2126-2136.

Jacob Devlin, Ming-Wei Chang, Kenton Lee, and Kristina Toutanova. 2019. BERT: pre-training of deep bidirectional transformers for language understanding. In Proceedings of the 2019 Conference of the North American Chapter of the Association for Computational Linguistics: Human Language Technologies, NAACL-HLT 2019, Minneapolis, MN, USA, June 2-7, 2019, Volume 1 (Long and Short Papers), pages 4171-4186. Association for Computational Linguistics.

Timothy Dozat and Christopher D Manning. 2016. Deep biaffine attention for neural dependency parsing. arXiv preprint arXiv:1611.01734.

Yanai Elazar, Shauli Ravfogel, Alon Jacovi, and Yoav Goldberg. 2020. When bert forgets how to pos: Amnesic probing of linguistic properties and mlm predictions.

Jessica Ficler and Yoav Goldberg. 2017. Controlling linguistic style aspects in neural language generation. arXiv preprint arXiv:1707.02633.

Zhenxin Fu, Xiaoye Tan, Nanyun Peng, Dongyan Zhao, and Rui Yan. 2018. Style transfer in text: Exploration and evaluation. In Thirty-Second AAAI Conference on Artificial Intelligence.

Leon A. Gatys. 2017. Texture synthesis and style transfer using perceptual image representations from convolutional neural networks. Ph.D. thesis, University of Tübingen, Germany.

Yoav Goldberg. 2019. Assessing BERT's syntactic abilities. CoRR, abs/1901.05287.

Kristina Gulordava, Piotr Bojanowski, Edouard Grave, Tal Linzen, and Marco Baroni. 2018. Colorless green recurrent networks dream hierarchically. In Proceedings of the Conference of the North American Chapter of the Association for Computational Linguistics: Human Language Technologies, NAACL-HLT, pages 1195-1205.

Naama Hadad, Lior Wolf, and Moni Shahar. 2018. A two-step disentanglement method. In IEEE Conference on Computer Vision and Pattern Recognition, (CVPR), pages 772-780.

John Hewitt and Percy Liang. 2019. Designing and interpreting probes with control tasks. In Proceedings of the 2019 Conference on Empirical Methods 
in Natural Language Processing and the 9th International Joint Conference on Natural Language Processing, EMNLP-IJCNLP 2019, Hong Kong, China, November 3-7, 2019, pages 2733-2743. Association for Computational Linguistics.

John Hewitt and Christopher D. Manning. 2019. A structural probe for finding syntax in word representations. In Proceedings of the Conference of the North American Chapter of the Association for Computational Linguistics: Human Language Technologies, NAACL-HLT, pages 4129-4138.

Elad Hoffer and Nir Ailon. 2015. Deep metric learning using triplet network. In Similarity-Based Pattern Recognition - Third International Workshop, SIM$B A D$, pages 84-92.

Matthew Honnibal and Mark Johnson. 2015. An improved non-monotonic transition system for dependency parsing. In Proceedings of the 2015 conference on empirical methods in natural language processing, pages 1373-1378.

Matthew Honnibal and Ines Montani. 2017. spacy 2: Natural language understanding with bloom embeddings, convolutional neural networks and incremental parsing. To appear, 7(1).

Zhiting $\mathrm{Hu}$, Zichao Yang, Xiaodan Liang, Ruslan Salakhutdinov, and Eric P. Xing. 2017. Toward controlled generation of text. In Proceedings of the 34th International Conference on Machine Learning, ICML 2017, Sydney, NSW, Australia, 6-11 August 2017, pages $1587-1596$.

Dieuwke Hupkes, Sara Veldhoen, and Willem $\mathrm{H}$. Zuidema. 2018. Visualisation and 'diagnostic classifiers' reveal how recurrent and recursive neural networks process hierarchical structure. J. Artif. Intell. Res., 61:907-926.

Mandar Joshi, Omer Levy, Daniel S Weld, and Luke Zettlemoyer. 2019. BERT for coreference resolution: Baselines and analysis. arXiv preprint arXiv:1908.09091.

Diederik P. Kingma and Jimmy Ba. 2015. Adam: A method for stochastic optimization. In International Conference on Learning Representations, ICLR.

Nikita Kitaev and Dan Klein. 2018. Constituency parsing with a self-attentive encoder. In Proceedings of the Annual Meeting of the Association for Computational Linguistics (ACL).

Guillaume Lample, Sandeep Subramanian, Eric Smith, Ludovic Denoyer, Marc'Aurelio Ranzato, and YLan Boureau. 2018. Multiple-attribute text rewriting. In International Conference on Learning Representations

Omer Levy and Yoav Goldberg. 2014. Linguistic regularities in sparse and explicit word representations. In Proceedings of the eighteenth conference on computational natural language learning, pages 171180
Xiang Lisa Li and Jason Eisner. 2019. Specializing word embeddings (for parsing) by information bottleneck. In Proceedings of the Conference on Empirical Methods in Natural Language Processing (EMNLP).

Yongjie Lin, Yi Chern Tan, and Robert Frank. 2019. Open sesame: Getting inside berts linguistic knowledge. In Proceedings of the 2019 ACL Workshop BlackboxNLP: Analyzing and Interpreting Neural Networks for NLP, pages 241-253.

Nelson F Liu, Matt Gardner, Yonatan Belinkov, Matthew Peters, and Noah A Smith. 2019a. Linguistic knowledge and transferability of contextual representations. arXiv preprint arXiv:1903.08855.

Yinhan Liu, Myle Ott, Naman Goyal, Jingfei Du, Mandar Joshi, Danqi Chen, Omer Levy, Mike Lewis, Luke Zettlemoyer, and Veselin Stoyanov. 2019b. RoBERTa: A robustly optimized BERT pretraining approach. CoRR, abs/1907.11692.

Laurens van der Maaten and Geoffrey Hinton. 2008. Visualizing data using t-SNE. Journal of Machine Learning Research, 9:2579-2605.

Michaël Mathieu, Junbo Jake Zhao, Pablo Sprechmann, Aditya Ramesh, and Yann LeCun. 2016. Disentangling factors of variation in deep representation using adversarial training. In Advances in Neural Information Processing Systems, pages 5041-5049.

Rowan Hall Maudslay, Josef Valvoda, Tiago Pimentel, Adina Williams, and Ryan Cotterell. 2020. A tale of a probe and a parser. CoRR, abs/2005.01641.

Tomas Mikolov, Ilya Sutskever, Kai Chen, Greg S Corrado, and Jeff Dean. 2013a. Distributed representations of words and phrases and their compositionality. In Advances in neural information processing systems, pages 3111-3119.

Tomas Mikolov, Wen-tau Yih, and Geoffrey Zweig. 2013b. Linguistic regularities in continuous space word representations. In Proceedings of the 2013 Conference of the North American Chapter of the Association for Computational Linguistics: Human Language Technologies, pages 746-751.

Siddharth Narayanaswamy, Brooks Paige, Jan-Willem van de Meent, Alban Desmaison, Noah D. Goodman, Pushmeet Kohli, Frank D. Wood, and Philip H. S. Torr. 2017. Learning disentangled representations with semi-supervised deep generative models. In Advances in Neural Information Processing Systems, pages 5925-5935.

Xi Peng, Xiang Yu, Kihyuk Sohn, Dimitris N. Metaxas, and Manmohan Chandraker. 2017. Reconstructionbased disentanglement for pose-invariant face recognition. In IEEE International Conference on Computer Visionn (ICCV), pages 1632-1641. 
Jeffrey Pennington, Richard Socher, and Christopher Manning. 2014. Glove: Global vectors for word representation. In Proceedings of the 2014 conference on empirical methods in natural language processing (EMNLP), pages 1532-1543.

Matthew E. Peters, Mark Neumann, Mohit Iyyer, Matt Gardner, Christopher Clark, Kenton Lee, and Luke Zettlemoyer. 2018. Deep contextualized word representations. In Proceedings of the 2018 Conference of the North American Chapter of the Association for Computational Linguistics: Human Language Technologies, NAACL-HLT 2018, New Orleans, Louisiana, USA, June 1-6, 2018, Volume 1 (Long Papers), pages 2227-2237. Association for Computational Linguistics.

Abhilasha Ravichander, Yonatan Belinkov, and Eduard H. Hovy. 2020. Probing the probing paradigm: Does probing accuracy entail task relevance? CoRR, abs/2005.00719.

Emily Reif, Ann Yuan, Martin Wattenberg, Fernanda B Viegas, Andy Coenen, Adam Pearce, and Been Kim. 2019. Visualizing and measuring the geometry of bert. In Advances in Neural Information Processing Systems, pages 8592-8600.

Kyle Richardson, Hai Hu, Lawrence S Moss, and Ashish Sabharwal. 2019. Probing natural language inference models through semantic fragments. arXiv preprint arXiv:1909.07521.

Florian Schroff, Dmitry Kalenichenko, and James Philbin. 2015. FaceNet: A unified embedding for face recognition and clustering. In IEEE Conference on Computer Vision and Pattern Recognition $(C V P R)$.

Kihyuk Sohn, Honglak Lee, and Xinchen Yan. 2015. Learning structured output representation using deep conditional generative models. In Advances in neural information processing systems, pages 34833491 .

Ian Tenney, Dipanjan Das, and Ellie Pavlick. 2019a. BERT rediscovers the classical NLP pipeline. In Proceedings of the Conference of the Association for Computational Linguistics, ACL, pages 4593-4601.

Ian Tenney, Patrick Xia, Berlin Chen, Alex Wang, Adam Poliak, R Thomas McCoy, Najoung Kim, Benjamin Van Durme, Sam Bowman, Dipanjan Das, and Ellie Pavlick. 2019b. What do you learn from context? probing for sentence structure in contextualized word representations. In International Conference on Learning Representations.

Naftali Tishby, Fernando C Pereira, and William Bialek. 1999. The information bottleneck method. In Proc. of the Allerton Allerton Conference on Communication, Control and Computing.

Marten van Schijndel and Tal Linzen. 2018. Modeling garden path effects without explicit hierarchical syntax. In Proceedings of the 40th Annual Conference of the Cognitive Science Society, pages 2600-2605. Cognitive Science Society.

Ethan Wilcox, Roger Levy, Takashi Morita, and Richard Futrell. 2018. What do RNN language models learn about filler-gap dependencies? In Proceedings of the EMNLP Workshop BlackboxNLP: Analyzing and Interpreting Neural Networks for NLP, pages 211-221. Association for Computational Linguistics.

Wei Yang, Yuqing Xie, Aileen Lin, Xingyu Li, Luchen Tan, Kun Xiong, Ming Li, and Jimmy Lin. 2019. End-to-end open-domain question answering with BERTserini. In Proceedings of the Conference of the North American Chapter of the Association for Computational Linguistics NAACL-HLT, pages 72 77. 


\section{A Additional Query-Value Examples}

- Q: as they did, the probability of an impact event temporarily climbed, peaking at 2 . $\mathrm{N}$ : however, the probability of flipping a head after having already flipped 20 heads in a row is simply

NT: during the first year, the scope of red terror expanded significantly and the number of executions grew into the thousands .

- Q: the celtics honored his memory during the following season by retiring his number 35 .

$\mathrm{N}$ : the beatles performed the song at the 1969 let it be sessions .

NT: the warriors dedicated their round five home match to fai 's memory.

- Q: in the old zurich war, the swiss confederation plundered the monastery, whose monks had fled to zurich.

$\mathrm{N}$ : the hridaya stra and the "five meditations" are recited, after which monks will be served with the gruel and vegetables.

NT: other commanders were killed and later rooplo kolhi was arrested near pag wool well , where his troops were fetching water.

- Q: the main cause of the punic wars was the conflict of interests between the existing carthaginian empire and the expanding roman republic.

$\mathrm{N}$ : the main issue was whether or not something had to be directly perceptible (meaning intelligible to an ordinary human being ) for it to be a " copy .

NT: the main enemy of the game is a sadistic but intelligent arms-dealer known as the jackal, whose guns are fueling the violence in the country.

- Q: jones maintained lifelong links with his native county, where he had a home, bron menai, dwyran.

$\mathrm{N}$ : his association with the bbc ended in 1981 with a move back to his native county and itv company yorkshire television, replacing martin tyler as the regional station 's football commentator.

NT: he leaves again for his native england, moving to a place near bath, where he works with a powerful local coven .
- Q: silver iodate can be obtained by reacting silver nitrate ( agno3) with sodium iodate. $\mathrm{N}$ : best mechanical strength is obtained if both sides of the disc are fused to the same type of glass tube and both tubes are under vacuum.

NT: each of these options can be obtained with a master degree from the university along with the master of engineering degree .

- Q: it confirmed that thomas medwin was a thoroughly learned man, if occasionally imprecise and careless

$\mathrm{N}$ : it was confirmed that the truth about heather's murder would be revealed which ultimately led to ben 's departure.

NT: it proclaimed that the entire movement of plastic art of our time had been thrown into confusion by the discoveries above-mentioned

- Q: after the death of nadab and abihu, moses dictated what was to be done with their bodies

$\mathrm{N}$ : most sources indicate that while no marriage took place between haile melekot and woizero ijigayehu, sahle selassie ordered his grandson legitimized .

NT: vvkj pilots who flew the hurricane conversion considered it to be superior to the standard model .

- Q: letters were delivered to sorters who examined the address and placed it in one of a number of "pigeon holes".

$\mathrm{N}$ : $i$ examined and reported on the thread called transcendental meditation which appears on the page you linked to .

NT: ronson visits purported psychopaths, as well as psychologists and psychiatrists who have studied them, and meets with robert $d$.

- Q: slowboat to hades is a compilation dvd by gorillaz, released in october 2006.

$\mathrm{N}$ : the album was released in may 2003 as a single album with a bonus $\boldsymbol{d} \boldsymbol{v d}$.

NT: master series is a compilation album by the british synthpop band visage released in 1997.

- Q: however, there are also many theories and conspiracies that describe the basis of the plot 
$\mathrm{N}$ : the name tabasco is not definitively known with a number of theories debated among linguists .

NT: it is likely that to this day there are some harrisons and harrises that are related.

- Q: nne, married first, to richard, eldest son of sir richard nagle, secretary of state for ireland, temp.

$\mathrm{N}$ : in the early 1960s, profumo was the secretary of state for war in harold macmillan's conservative government and was married to actress valerie hobson .

NT: he was born in edinburgh, the son of william simpson, minister of the tron church , edinburgh, by his wife jean douglas balderston .

- Q: battle of stoke field, the final engagement of the wars of the roses.

$\mathrm{N}$ : among others, hogan announced the "engagement " of utah-born pitcher roy castleton

NT: song of susannah, the sixth installment in the dark tower series.

- Q: it vies for control with its host, causing physiological changes that will eventually cause the host 's internal organs to explode.

$\mathrm{N}$ : hurtig and loewen developed rival factions within the party, and battled for control .

NT: players take control of each of the four main characters at different times throughout the game, which enables multilateral perspective on the storyline .

- Q: as such, radio tirana kept close to the official policy of the people 's republic of china, which was also both anti-west and anti-soviet whilst still being socialist in tone.

$\mathrm{N}$ : this was in line with the policy outlined by constantine vii porphyrogenitus in de administrando imperio of fomenting strife between the rus' and the pechenegs .

NT: april 2006, the upr periodically examines the human rights performance of all 193 un member states .

- Q: the engine was designed to accept either regular grade, 87 octane gasoline or premium grade, 91 octane gasoline .

$\mathrm{N}$ : for example, an advanced html editing field could accept a pasted or inserted image and convert it to a data uri to hide the complexity of external resources from the user.

NT: it uses plug-ins ( html parsing technology ) to collect bibliographic information, videos and patents from webpages .

- Q: one such decree was the notorious 1876 ems ukaz, which banned the kulishivka and imposed a russian orthography until 1905 ( called the yaryzhka, after the russian letter yery).

$\mathrm{N}$ : fin 1612 , the shogun declared a decree that specifically banned the killing of cattle.

NT: tannis has eliminated the other time lords and set the doctor and the minister against each other .

- Q: a 25 degree list was reduced to 15 degrees; men had abandoned ship prematurely - hence the pow.

$\mathrm{N}$ : $i$ suggest the article be reduced to something over half the size .

NT: the old high school was converted into a middle school, until in 1971 the 5.

- Q: the library catalog is maintained on a database that is made accessible to users through the internet.

$\mathrm{N}$ : this screenshot is made for educational use and used for identification purposes in the article on nba on abc .

NT: hpc is the main ingredient in cellugel which is used in book conservation.

- Q: although he lost, he was evaluated highly by kazuyoshi ishii, and he was invited to seidokaikan .

$\mathrm{N}$ : he attended suny fredonia for one year and in 1976 received $a b$.

NT: played primarily as a small forward, he showed some opportunist play and in his 18 games managed a creditable 12 goals .

- Q: for each round won, you gain one point towards winning the match .

$\mathrm{N}$ : in the fourth round, federer beat tommy robredo and equalled jimmy connors' record of 27 consecutive grand slam quarterfinals . NT: at the beginning of each mission, as well as the end of the last mission, a cutscene is played that helps develop the story. 


\section{B BERT Closest-Word Results}

In Table 3, we present the full quantitative results when using BERT as the encoder. "Baseline" refers to unmodified vectors derived from BERT, and "Transformed" refers to the vectors after the learned syntactic transformation $f$. "hard" refers to evaluation on the subset of POS tags which are most structurally diverse.

\section{Complete Parsing Results}

Below are the LAS and UAS scores for the experiments described in $\S 4$.

\section{Examples of Equivalent Sentences}

In Table 6 we present randomly selected examples of groups of structurally-similar sentences $(\S 3.1)$. 


\begin{tabular}{|c|c|c|c|c|c|c|c|}
\hline & Dep. edge & Head's dep. edge & $\begin{array}{c}\text { Tree path } \\
\text { (complete) }\end{array}$ & $\begin{array}{c}\text { Tree path } \\
(\mathrm{L}=3)\end{array}$ & $\begin{array}{c}\text { Tree path } \\
(\mathrm{L}=2)\end{array}$ & $\begin{array}{c}\text { Depth } \\
\text { (correlation) }\end{array}$ & Lexical Match \\
\hline Baseline (all) & 0.549 & 0.432 & 0.146 & 0.310 & 0.522 & 0.436 & 0.829 \\
\hline Transformed (all) & 0.681 & 0.565 & 0.221 & 0.471 & 0.697 & 0.597 & 0.319 \\
\hline Baseline (hard) & 0.478 & 0.429 & 0.143 & 0.310 & 0.521 & 0.428 & 0.820 \\
\hline Transformed (hard) & 0.652 & 0.565 & 0.225 & 0.482 & 0.714 & 0.601 & 0.300 \\
\hline
\end{tabular}

Table 3: Full quantitative results when using BERT as the encoder. "Baseline" refers to unmodified vectors derived from BERT, and "Transformed" refers to the vectors after the learned syntactic transformation $f$. "hard" refers to evaluation on the subset of POS tags which are most structurally diverse.

\begin{tabular}{lrrrr}
\hline Model & \multicolumn{4}{c}{ Number of sentences } \\
& 50 & 100 & 200 & 500 \\
\hline POS & 0.60 & 0.65 & 0.71 & 0.75 \\
ELMO & 0.52 & 0.64 & 0.75 & 0.82 \\
ELMO-reduced & 0.55 & 0.65 & 0.75 & 0.82 \\
ELMO-PCA & 0.55 & 0.65 & 0.73 & 0.79 \\
ELMO-syntax (ours) & 0.61 & 0.70 & 0.76 & 0.83
\end{tabular}

Table 4: Labeled parsing scores (LAS)

\begin{tabular}{lrrrr}
\hline Model & \multicolumn{4}{c}{ Number of sentences } \\
& 50 & 100 & 200 & 500 \\
\hline POS & 0.66 & 0.71 & 0.77 & 0.80 \\
ELMO & 0.60 & 0.71 & 0.81 & 0.87 \\
ELMO-reduced & 0.68 & 0.76 & 0.83 & 0.88 \\
ELMO-PCA & 0.63 & 0.72 & 0.79 & 0.85 \\
ELMO-syntax (ours) & 0.69 & 0.78 & 0.82 & 0.87
\end{tabular}

Table 5: Unlabeled parsing scores (UAS) 


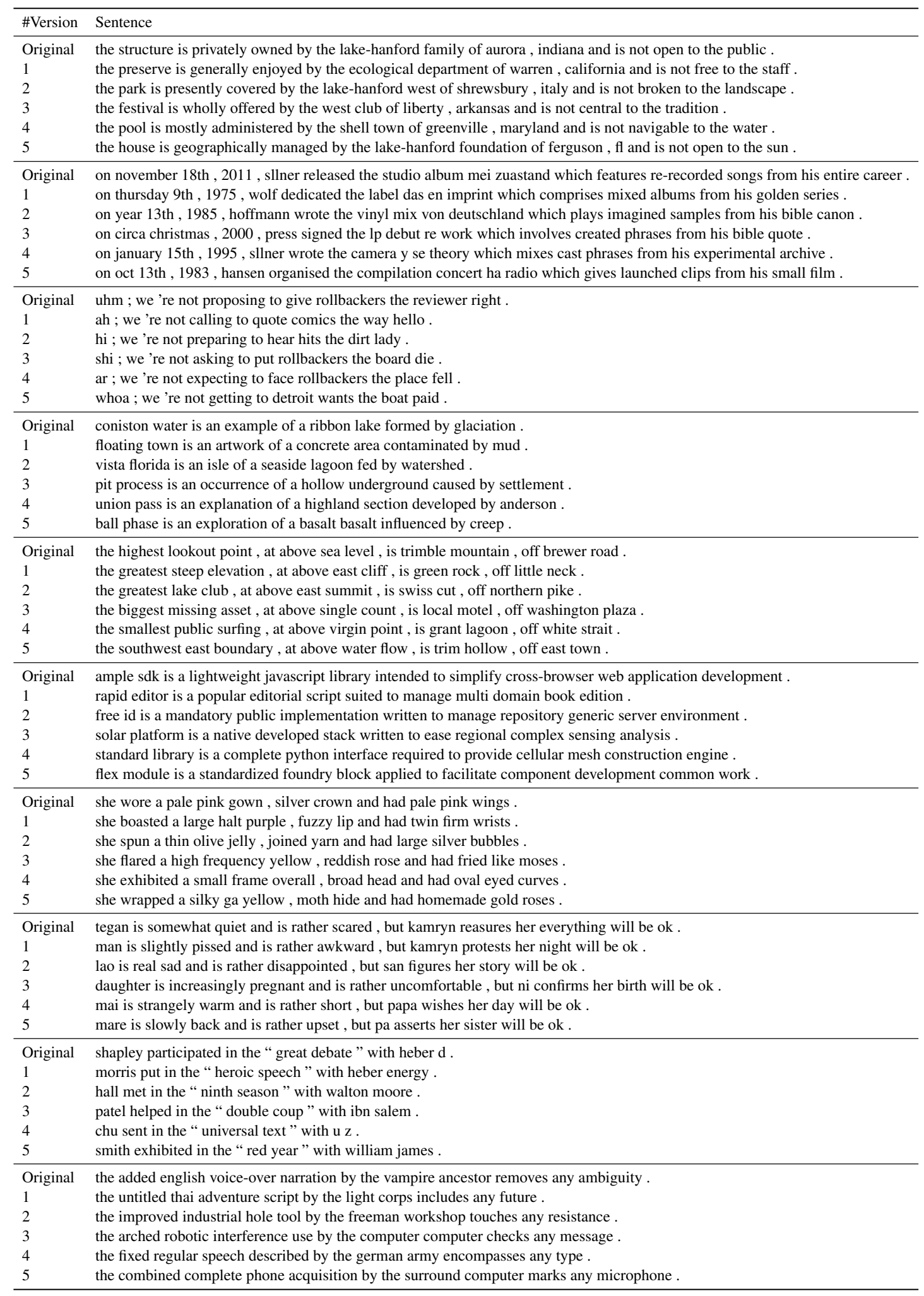

Table 6: Randomly selected examples of groups of structurally-similar sentences (§3.1) 